\title{
Acute erythroid leukemia: autopsy report of a rare disease
}

\author{
Cristiane Rúbia Ferreira ${ }^{a}$, Fabiana Roberto Lima ${ }^{a}$, Edna Harumi Goto \\ Elizabeth In Myung Kimº ${ }^{\mathrm{b}}$ Luciana Andréa Avena Smeilib, \\ Fernando Peixoto Ferraz de Campos ${ }^{\mathrm{b}}$, Maria Claudia Nogueira Zerbinic
}

Ferreira CR, Lima FR, Goto EH, et al. Acute erythroid leukemia: autopsy report of a rare disease. Autopsy Case Rep [Internet]. 2011;1(4):11-20. http://dx.doi.org/10.4322/acr.2011.012

\section{ABSTRACT}

Acute erythroid leukemia (AEL) is a rare subtype of acute myeloid leukemia (AML), characterized by predominant erythroid proliferation. The 2008 World Health Organization (WHO) classification of AML defined two AEL subtypes: erythroleukaemia (EL), in which erythroid precursors account for $50 \%$ or more of all nucleated bone marrow cells and myeloblasts account for $20 \%$ or more of the nonerythroid cell population; and pure erythroid leukemia (PEL), in which erythroid precursors account for $80 \%$ or more of all nucleated bone marrow cells. We report the case of an elderly female patient with wasting syndrome and pancytopenia without evidence of blasts in peripheral blood. A diagnosis of PEL was established on the basis of bone marrow biopsy findings. The patient died on postadmission day 20 , and an autopsy was performed. We reclassified the disease as EL on the basis of the autopsy findings, which included myeloblasts accounting for more than $20 \%$ of the nonerythroid cells in the bone marrow, as well as leukemic infiltration and myeloid metaplasia in solid organs, such as the liver, spleen, kidneys, adrenal glands, and abdominal lymph nodes. A rare disease, AEL accounts for less than $5 \%$ of all AMLs and is practically a diagnosis of exclusion. Autopsy reports of AEL are extremely rare in the literature. We demonstrate that in the case reported here, leukemia cells tended to infiltrate solid organs with myeloid metaplasia. Our findings also show that a larger neoplastic bone marrow sample is crucial to the correct diagnosis of EL, which is based on morphological and quantitative criteria.

Keywords: Leukemia; Leukemia erythroblastic acute; Autopsy.

\section{CASE REPORT}

\section{Clinical Case}

An 86-year-old Black female patient sought medical attention with a 1-month history of generalized muscle weakness, dyspnea on minimal exertion, adynamia, apathy, loss of appetite, and depressed mood, all of which coincided with the death of a son. She had also had episodes of diarrhea accompanied by weight loss, having lost approximately $5 \mathrm{~kg}$ during that same period.

\footnotetext{
a Anatomic Pathology Service - Hospital Universitário, Universidade de São Paulo, São Paulo/SP - Brazil.

${ }^{b}$ Department of Internal Medicine - Hospital Universitário, Universidade de São Paulo, São Paulo/SP - Brazil.
}

Copyright $\odot 2011$ Autopsy and Case Reports - This is an Open Access article distributed of terms of the Creative Commons Attribution NonCommercial License (http://creativecommons.org/licenses/by/3.0/) which permits unrestricted non-commercial use, distribution, and reproduction in any médium provided article is properly cited. 
The patient reported that she had been diagnosed with anemia of unknown etiology 6 years prior, having once received blood transfusion but never having been treated with erythropoietin. She stated that she had never been diagnosed with or treated for any type of neoplasia. She had a history of diabetes mellitus (DM) and hypothyroidism, as well as a more than 30-year history of arterial hypertension (for which she had been under clinical treatment), together with a history of coronary heart disease, having undergone myocardial revascularization 7 years prior. She had been under treatment with metformin, gliclazide, levothyroxine, enalapril, amlodipine, cilostazol, and sertraline. The patient had been smoking hand-rolled cigarettes for 30 years.

Physical examination at admission revealed normal respiration, pallor, poor general health status, and a body weight of $46 \mathrm{~kg}$. She presented with a heart rate of $72 \mathrm{bpm}$, a respiratory rate of 20 breaths/minutes, and a blood pressure of $134 / 56 \mathrm{mmHg}$. In addition, she presented with no edema, jugular stasis, or peripheral lymph node enlargement. Endoscopy revealed oral candidiasis. Although pulmonary auscultation revealed no abnormalities, cardiac auscultation revealed rhythmic heart sounds with aortic and mitral systolic murmur. The abdomen was normal, and abdominal palpation revealed no organomegaly. Neurological examination revealed no abnormalities. The clinical investigation was based on a working diagnosis of wasting syndrome, with a focus on hematologic malignancy and solid tumors.

During her hospital stay, the patient presented with loss of appetite, frequent nausea, diarrhea, and hematochezia. Despite low food intake, there was no additional weight loss. Sigmoidoscopy revealed moderate proctitis with signs of bleeding. During the sigmoidoscopy, a rectal biopsy was performed. Histopathological examination of the biopsy specimen revealed mild inflammatory infiltrate composed of lymphomononuclear cells in the superficial layer of the colonic mucosa, with reactive intestinal crypts. The results of the laboratory tests performed are shown in Table 1.

A blood workup was performed and revealed normocytic anemia, thrombocytopenia, and leukoerythroblastic reaction. Urinalysis revealed mild proteinuria with hyaline and granular casts. Serology for HIV, hepatitis $B$, and hepatitis $C$ was negative. Serum levels of vitamin B12, folic acid, and iron were normal, as were those of the tumor markers carcinoembryonic antigen, cancer antigen 125 , and cancer antigen 19-9. Upper gastrointestinal endoscopy revealed erosive antral gastritis. A computed tomography (CT) scan of the abdomen and pelvis revealed mild splenomegaly and atheromatous aorta. A CT scan of the chest revealed atheromatous aorta, normal lung parenchyma, and no lymph node enlargement. An echocardiogram showed mild left

Table 1 - Laboratory test results

\begin{tabular}{|c|c|c|c|c|c|c|c|}
\hline Test & Measure & Value & Reference & Test & Measure & Value & Reference \\
\hline Hemoglobin & g.dL ${ }^{-1}$ & 9.7 & $12.3-15.3$ & Creatinine & $\mathrm{mg} \cdot \mathrm{dL}^{-1}$ & 2.2 & $0.4-1.3$ \\
\hline Hematocrit & $\%$ & 31 & $36.0-45.0$ & Urea & $\mathrm{mg} \cdot \mathrm{dL}^{-1}$ & 121 & $10-50$ \\
\hline MCV & $\mathrm{fl}$ & 85 & $80-96$ & Ionized calcium & $\mathrm{mmol} \cdot \mathrm{L}^{-1}$ & 1.14 & $1.15-1.35$ \\
\hline RDW & $\%$ & 16.1 & $11-16$ & Sodium & $\mathrm{mEq} \cdot \mathrm{L}^{-1}$ & 132 & $136-146$ \\
\hline Leukocytes & $\mathrm{mm}^{3}$ & 9,000 & $4.4-11.3 \times 10^{3}$ & Potassium & $\mathrm{mEq} \cdot \mathrm{L}^{-1}$ & 4.2 & $3.5-5.0$ \\
\hline Myelocytes & $\%$ & 2 & 0 & AST & U.L $L^{-1}$ & 20 & $10-31$ \\
\hline Metamyelocytes & $\%$ & 1 & 0 & ALT & $U . L^{-1}$ & 15 & $9-36$ \\
\hline Band neutrophils & $\%$ & 22 & $1-5$ & ALP & U. $L^{-1}$ & 1445 & $10-100$ \\
\hline Segmented neutrophils & $\%$ & 55 & $46-75$ & Total bilirubin & $\mathrm{mg} \cdot \mathrm{dL}^{-1}$ & 0.3 & $0.3-1.2$ \\
\hline Eosinophils & $\%$ & 0 & $1-4$ & Total proteins & $\mathrm{g} \cdot \mathrm{dL}^{-1}$ & 5.8 & $6.0-8.0$ \\
\hline Basophils & $\%$ & 0 & $0-2.5$ & Albumin & g.dL ${ }^{-1}$ & 2.5 & $3.0-5.0$ \\
\hline Lymphocytes & $\%$ & 12 & $18-40$ & $\mathrm{DHL}$ & $U . L^{-1}$ & 3300 & $120-246$ \\
\hline Monocytes & $\%$ & 8 & $2-9$ & $\mathrm{TSH}$ & $\mu U \mathrm{UI} . \mathrm{L}^{-1}$ & 2.55 & $0.55-4.78$ \\
\hline Platelets & $\mathrm{mm}^{3}$ & 80,000 & $150-400 \times 10^{3}$ & Prothrombin time & INR & 1.23 & 1 \\
\hline
\end{tabular}

MCV, mean corpuscular volume; RDW, red cell distribution width; AST, aspartate aminotransferase; ALT, alanine aminotransferase; ALP, alkaline phosphatase; TSH, thyroid stimulating factor; INR, international normalized ratio. 
ventricular hypertrophy, inferior wall hypokinesia, mitral insufficiency, and aortic stenosis.

There was a progressive decrease in leukocyte and platelet counts (which dropped to 1,100 and 5,000 cells. $\mathrm{mm}^{-3}$, respectively), accompanied by a worsening of renal function. After a failed bone marrow (BM) aspiration (i.e., a "dry tap"), the patient underwent BM biopsy, which revealed extensive infiltration of the BM by aggregates of large, atypical cells (approximately $70 \%$ ), the immunophenotype of which was consistent with that of cells of the erythroid lineage (positive for glycophorin A and partially positive for CD117); other findings included extensive areas of necrosis, numerous mitotic figures, and markedly reduced BM reserve (Figure 1). Those findings were consistent with a diagnosis of pure erythroid leukemia (PEL), in accordance with the World Health Organization (WHO) classification of acute myeloid leukemia (AML), ${ }^{1}$ or $\mathrm{M} 6$, in accordance with the French-American-British (FAB) classification. ${ }^{2}$

Despite the supportive treatment administered, the patient presented with progressive worsening of overall health status, decreased hemoglobin levels (having received a transfusion of packed red cells), dyspnea, abdominal distension, and altered level of consciousness. The patient died on postadmission day 20 , and an autopsy was performed.

\section{Autopsy Findings}

The abdominal and thoracic cavities were opened, revealing no ectopia or effusion. There was mild hepatomegaly, the liver weighing $2,100 \mathrm{~g}$ (reference value, 1,400-1,600 g). On sectioning, the liver parenchyma had a mottled appearance, suggestive of passive congestion, with no evidence of nodular areas. The spleen showed a slight increase in volume (weight, $239 \mathrm{~g}$; reference value, 150-250 g), the splenic parenchyma was homogeneous, as well as being wine-red on sectioning. The kidneys (right kidney weight, $180 \mathrm{~g}$; left kidney weight, $200 \mathrm{~g}$; reference value, $115-155 \mathrm{~g}$ ) had a granular outer surface and an appearance typical of renal vascular disease, with multiple retention cysts of less than $1.0 \mathrm{~cm}$ in diameter. In the right kidney, we found a solid, yellowish nodule of $1.0 \mathrm{~cm}$ in diameter in the cortical region. The heart (weight, $385 \mathrm{~g}$; reference value, $275 \pm 75 \mathrm{~g}$ ) showed fibrous adhesions to the pericardium. On sectioning, revealed left ventricular hypertrophy, the anterior wall of the left ventricle
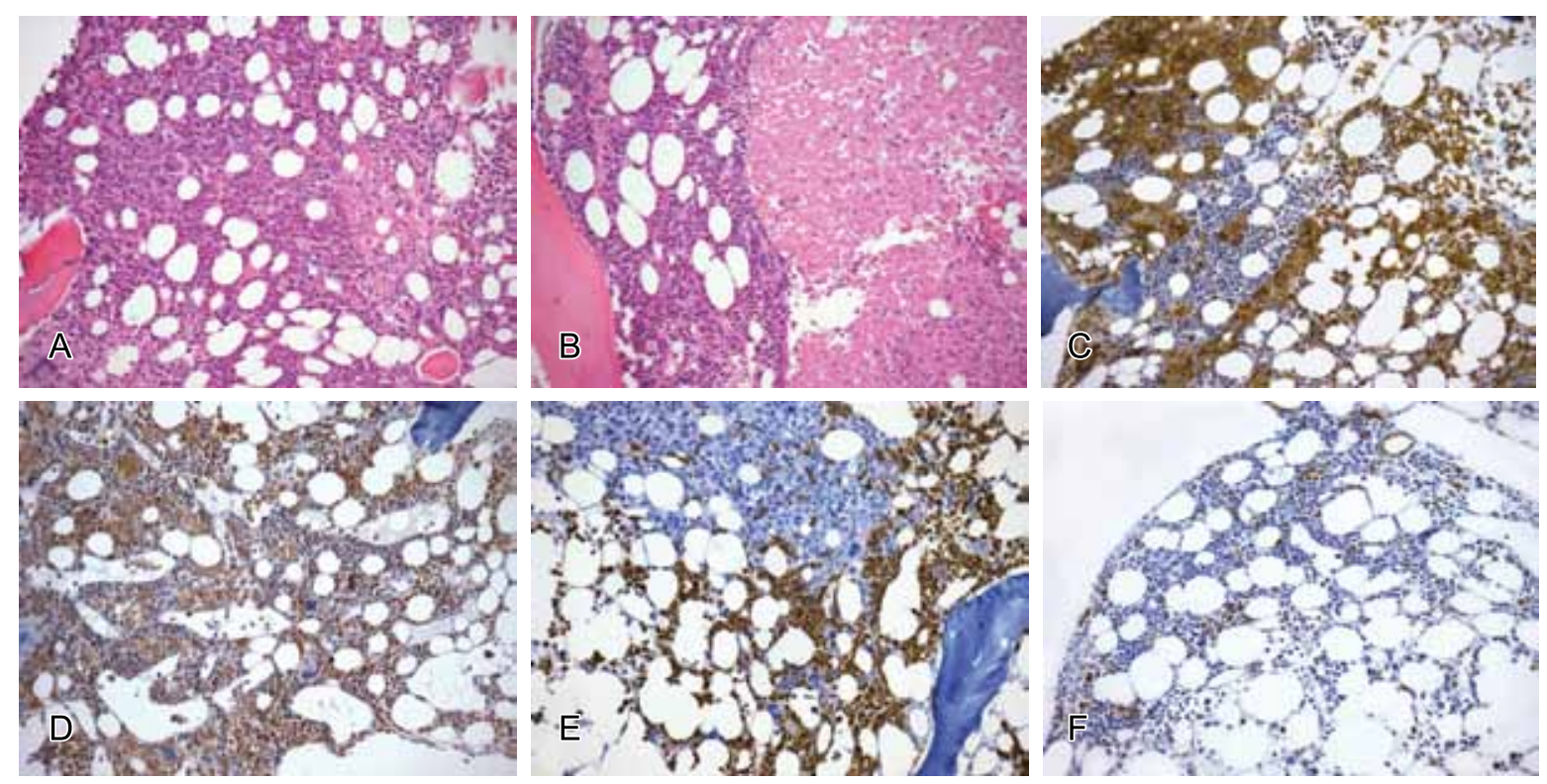

Figure 1 - Photomicrographs of bone marrow biopsy findings: A- bone marrow with increased cellularity and infiltration by immature erythroid cells (some binucleated) with voluminous nuclei, prominent nucleoli, and basophilic cytoplasm (hematoxylin and eosin [H\&E]; magnification, $\times 100$ ); $\mathbf{B}$ - extensive area of coagulative necrosis of neoplastic cells (H\&E; magnification, $\times 200$ ); C- immature erythroid cells diffusely positive for glycophorin A (immunohistochemistry [IHC]; magnification, $\times 100$ ); D- CD117-positive (IHC; magnification, $\times 200$ ); E- cells of the granulocytic series focally positive for myeloperoxidase (IHC; magnification, $\times 100$ ); and F- CD34 positivity in blasts (in the topography of myeloperoxidase-positive cells) and in small vessels (IHC; magnification, $\times 400)$. 

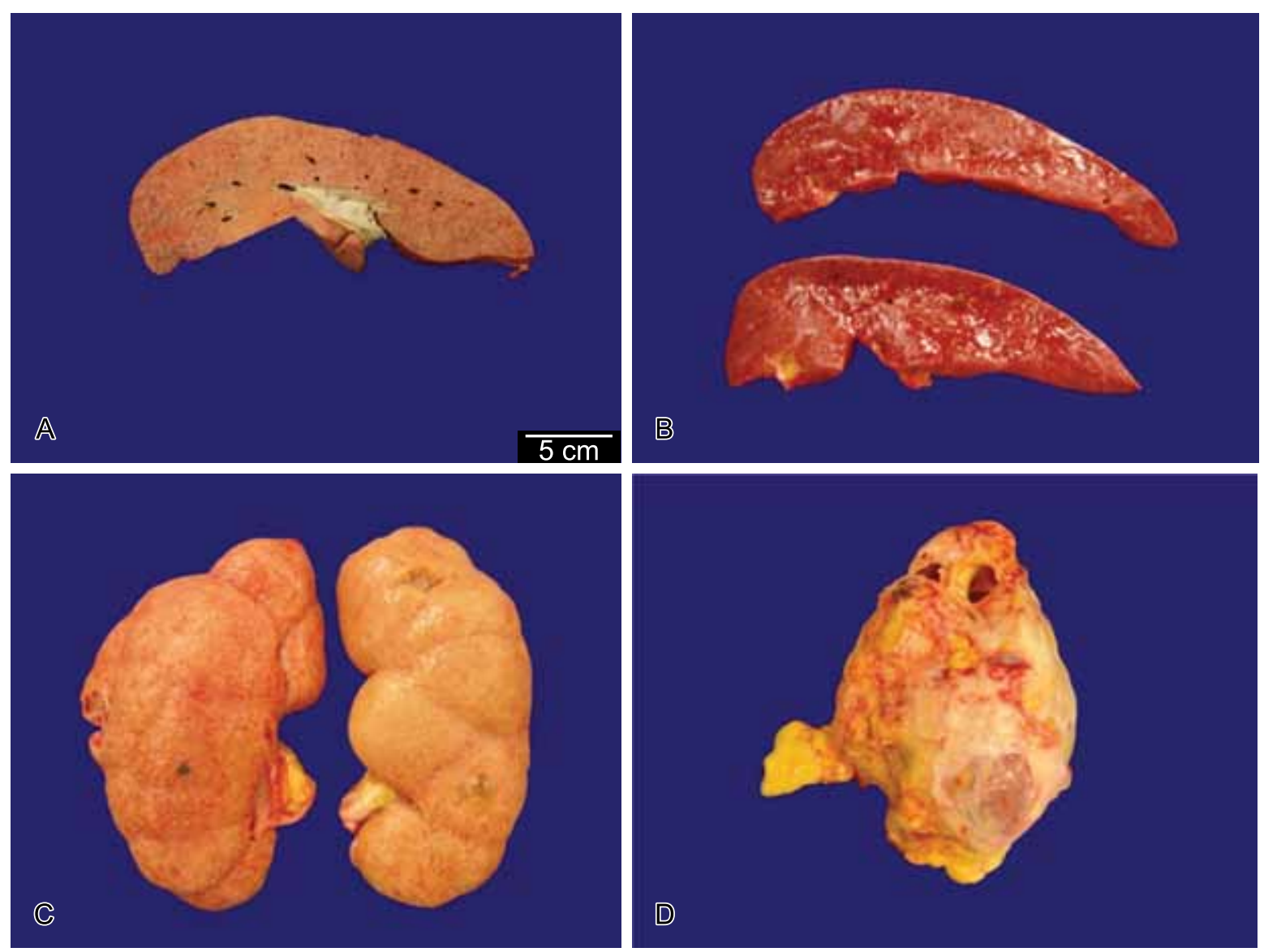

Figure 2 - Panoramic view of solid organs and the heart: A- liver parenchyma with a mottled appearance, suggestive of passive congestion; B- homogeneous, wine-red splenic parenchyma; $\mathbf{C}$ - right and left kidneys with granular surface and retention cysts; and D- heart, showing fibrous adhesions to the pericardium.

showing a whitish area, which was suggestive of prior myocardial infarction (Figure 2).

In the right lung (right lung weight, $355 \mathrm{~g}$; reference value, $450 \mathrm{~g}$-left lung weight, $295 \mathrm{~g}$; reference value, $375 \mathrm{~g}$ ), we identified a small, redwine colored nodule measuring $0.5 \mathrm{~cm}$ in diameter. We opened the gastrointestinal tract and found hyperemia of the rectal mucosa. The aorta was affected by severe atherosclerosis. No uterus was found.

Microscopic examination of the BM from iliac crests and right and left ribs revealed extensive necrotic areas, interspersed with areas of infiltration by atypical undifferentiated cells with large nuclei and prominent nucleoli, sometimes binucleated and had a basophilic cytoplasm (about $80 \%$ of the nucleated cells). Residual hematopoietic tissue was represented by cells of the granulocytic lineage, with evident delay in maturation and very few megakaryocytes. Immunohistochemical analysis revealed that the immunophenotype of the atypical undifferentiated cells was similar to that of those analyzed after the BM biopsy, i.e., glycophorin A-positive and CD117-positive, as well as CD34-negative. The immunophenotype of the granulocytic series was myeloperoxidase (MPO)positive and CD117-positive, as well as positive coexpression of CD34 (in 20-30\% of the nucleated cells), showing the presence of myeloblasts in more than $20 \%$ of the nonerythroid nucleated cells (Figure 3 ). The malignancy was reclassified as acute erythroid leukemia (AEL) of the erythroleukaemia (EL) subtype (also known as erythroid/myeloid), in accordance with the WHO classification. ${ }^{1}$

Immunohistochemistry revealed membrane positivity for E-cadherin in very few of the cells of the BM biopsy, namely in those that were large and immature (Figure 4). In contrast, E-cadherin immunohistochemistry examination of the BM sections obtained during the autopsy revealed no such evidence.

The solid organs, namely the liver, spleen, kidneys, adrenal glands, and abdominal lymph nodes, were found to be infiltrated by leukemia cells. The infiltration was accompanied by myeloid metaplasia, which was particularly evident in the spleen and liver. We also found severe megakaryocyte atypia, 

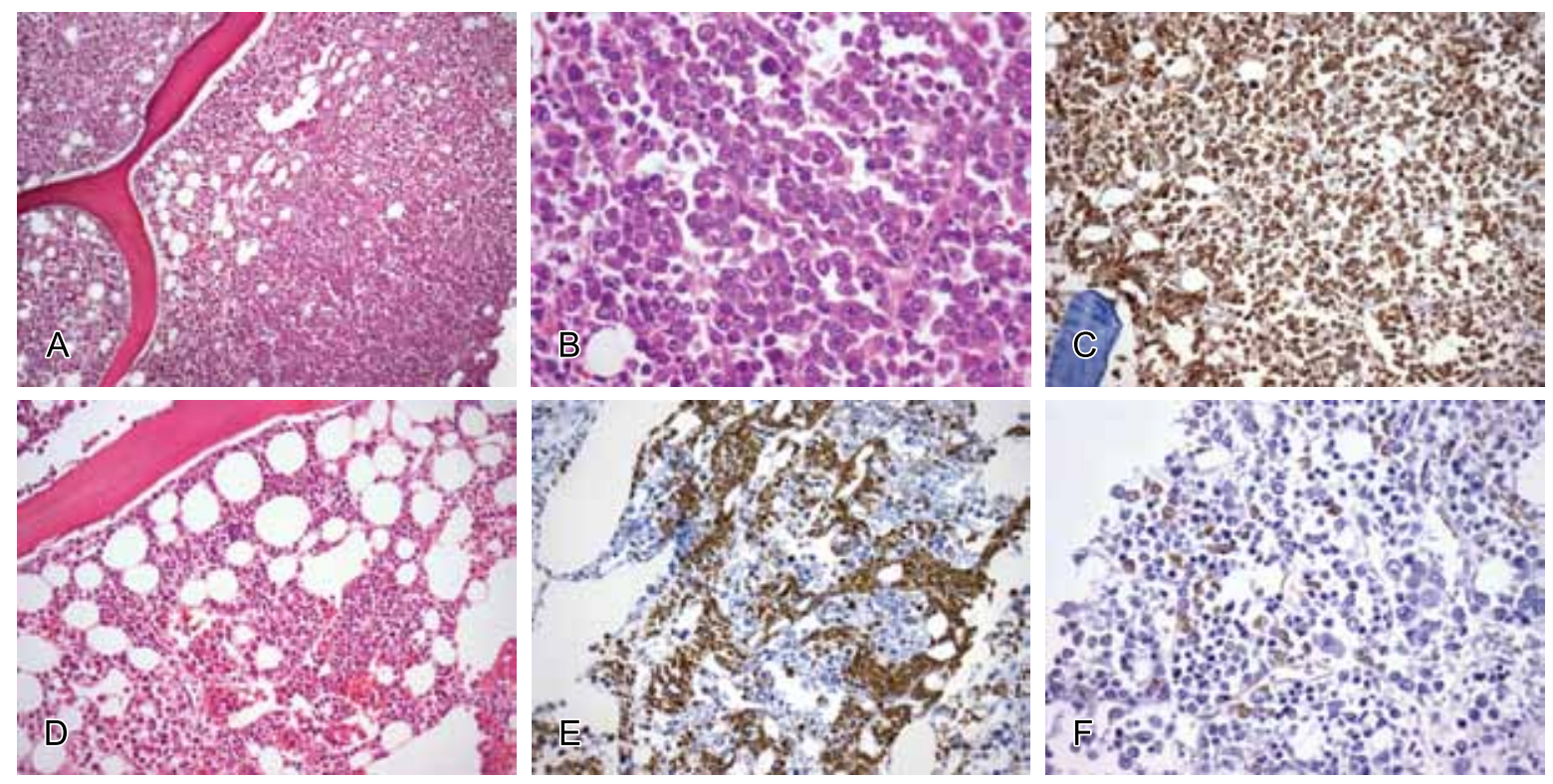

Figure 3 - Photomicrographs of autopsy findings in bone marrow: A- bone marrow with increased cellularity and infiltration by immature erythroid cells (hematoxylin and eosin [H\&E]; magnification, $\times 100$ ); B- immature erythroid cells (some binucleated) with voluminous nuclei, prominent nucleoli, and basophilic cytoplasm (H\&E; magnification, $\times 200$ ); C- immature erythroid cells diffusely positive for glycophorin A (immunohistochemistry [IHC]; magnification, $\times 100$ ); D- medium-sized immature myeloid cells within sinusoids (H\&E; magnification, $\times 200$ ); E-myeloperoxidase-positive immature myeloid cells predominantly within sinusoids (IHC; magnification, $\times 200$ ); and F- positivity for CD34 in blasts (in the topography of myeloperoxidase-positive cells) and in small vessels (IHC; magnification, $\times 400$ ).
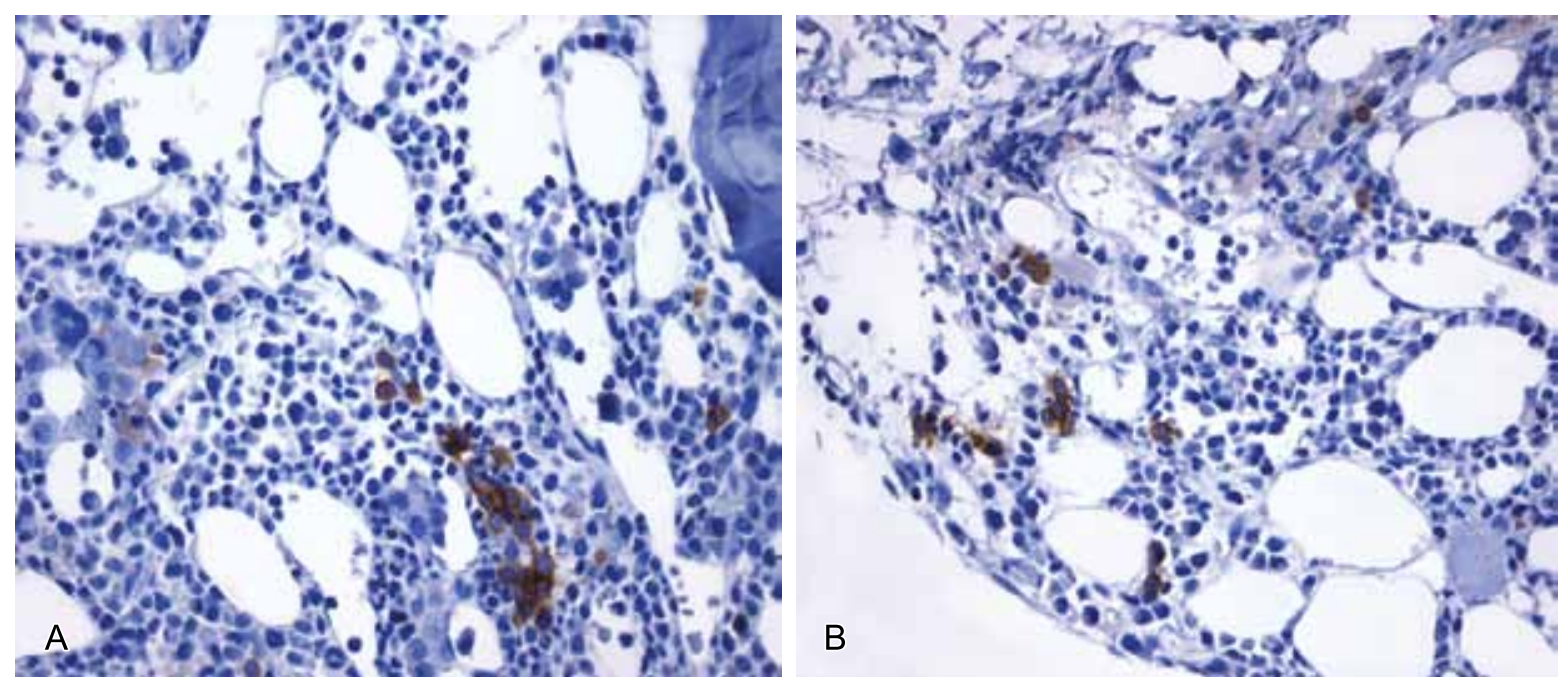

Figure 4 - Photomicrographs of bone marrow biopsy sample: A and B- very few cells (large and immature) showing positivity for E-cadherin (immunohistochemistry; magnification, $\times 400$ ).

including some with with multilobulated nuclei and coarse chromatin (Figures 5, 6, and 7).

The nodule in the right lung represented diffuse alveolar damage. In the renal parenchyma, we found retention cysts and mesangioproliferative glomerulonephritis secondary to DM, as well as a small renal clear cell carcinoma in the $1 \mathrm{~cm}$ right kidney nodule described above.

\section{DISCUSSION}

The pure erythroblast proliferation is very rare. AEL accounts for less than $5 \%$ of all AMLs. ${ }^{1}$ The diagnosis of AEL has been controversial since the initial concepts of the entity were first described by Di Guglielmo, in mid-1917. Di Guglielmo described two forms of the disease, namely erythremic myelosis (the more common form) and acute erythremia. ${ }^{2,3}$ In 

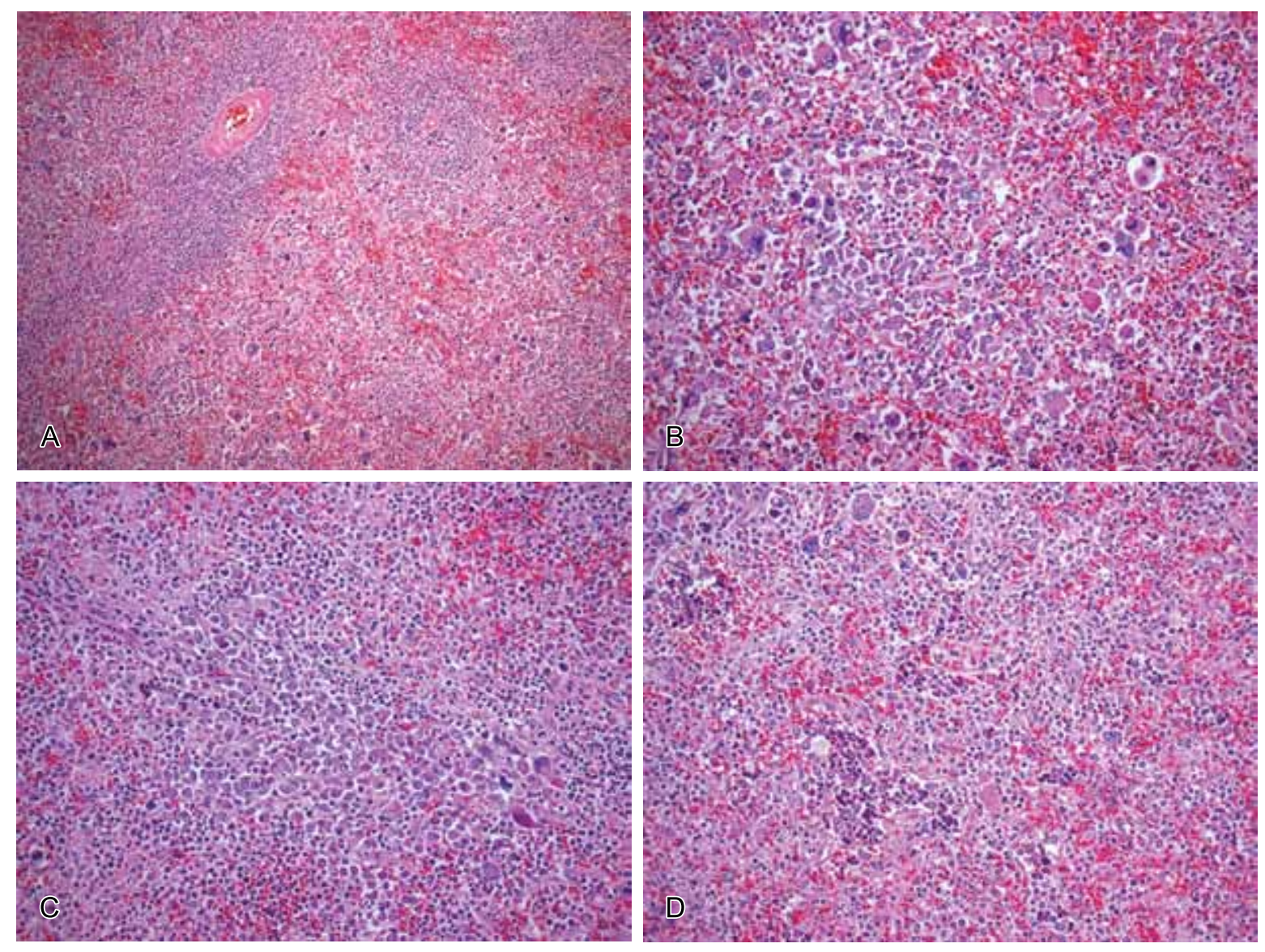

Figure 5- Photomicrographs of the spleen: A- red pulp of the spleen infiltrated by immature erythroid cells, with atypical megakaryocytes (hematoxylin and eosin [H\&E]; magnification, $\times 100$ ); B-immature erythroid cells, with atypical megakaryocytes (H\&E; magnification, $\times 200$ ); C- immature erythroid cells (some binucleated) with voluminous nuclei, prominent nucleoli, and basophilic cytoplasm (H\&E; magnification, $\times 200$ ); and D- red pulp of the spleen showing myeloid metaplasia, represented by atypical megakaryocytes and erythroid cell colonies (H\&E; magnification, $\times 200)$.

1976, and again in a revision made in 1985, the FAB Cooperative Group classified AEL as M6, a subtype of AML characterized by erythroblasts accounting for $50 \%$ or more of all BM hematopoietic cells and myeloblasts accounting for $30 \%$ or more of all nonerythroid cells. ${ }^{4,5}$ Some authors have informally expanded the category to include subtypes M6a (EL) and M6b (PEL). ${ }^{6-8}$ The 2001 WHO classification of AML officially recognized PEL by dividing AEL into two subtypes: EL (erythroid precursors accounting for at least $50 \%$ of the entire BM cell population and myeloblasts accounting for at least $20 \%$ of the nonerythroid cell population); and PEL (immature erythroid cells accounting for at least $80 \%$ of all BM cells, with no evidence of a significant myeloblastic component). ${ }^{9}$ Although the $2008 \mathrm{WHO}$ classification of AML was essentially the same as the previous classification, stricter criteria were established, entities such as AML with myelodysplasia-related changes (an entity that can present with erythroid predominance and that now comprises cases with $20 \%$ or more blasts and dysplasia involving at least $50 \%$ of cells of two or more lineages) and therapy-related AML having been created. ${ }^{1}$

We reported the case of an elderly female patient who presented with wasting syndrome and pancytopenia. On the basis of BM biopsy findings, we established an initial diagnosis of PEL. The analysis of a much larger BM sample during autopsy revealed that myeloblasts accounted for more than $20 \%$ of the nonerythroid cell population. This led us to establish a final diagnosis of EL. Although the understanding of AEL has increased over the years, the diagnostic criteria established in the AML classifications proposed by the FAB Cooperative Group ${ }^{4,5}$ and the $\mathrm{WHO}^{1,9}$ have always been based on morphological, quantitative, and immunophenotypic parameters, genetic markers having yet to be defined. ${ }^{6}$

The diagnostic criteria for AEL established in the latest $\mathrm{WHO}$ classification of $\mathrm{AML}^{1}$ are far stricter than were those established in previous 

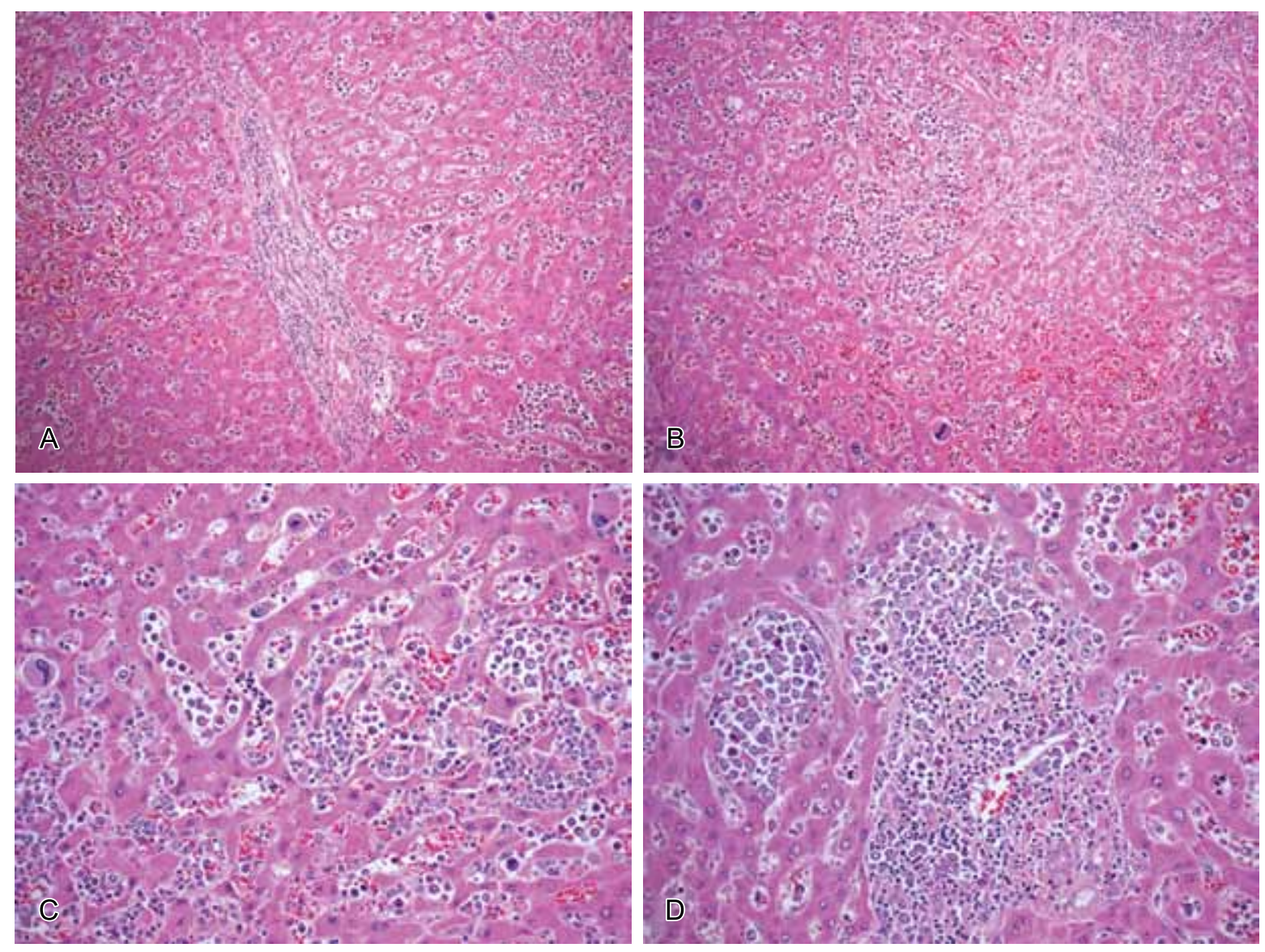

Figure 6 - Photomicrographs of the liver: A and B- liver parenchyma with leukemic infiltration in sinusoids and portal tracts (hematoxylin and eosin [H\&E]; magnification, $\times 100$ ); C- leukemic infiltration in hepatic sinusoids, with atypical megakaryocytes and focal myeloid metaplasia (H\&E; magnification, $\times 200$ ); and Dleukemic infiltration in portal tract, with immature erythroid cells (H\&E; magnification, $\times 200)$.

classifications. According to the current WHO criteria, a history of myelodysplastic syndromes, myeloproliferative disorders, exposure to toxins such as benzene, cancer chemotherapy, immunotherapy, radiation therapy, or erythropoietin therapy rules out AEL. ${ }^{1,6}$ Such cases are currently diagnosed as AML with myelodysplasia-related changes or as therapy-related AML; this has significantly reduced the number of cases of AEL, which has practically become a diagnosis of exclusion. ${ }^{1,10}$ Although our patient presented with multiple comorbidities, she had no history of cancer treatment or diagnosis of myelodysplastic syndromes, her 6-year history of chronic anemia being possibly related to her chronic kidney disease and DM.

The etiology of AEL is unknown, and its risk factors have yet to be identified. ${ }^{6}$ The disease principally affects adults (mean age, 50 years), manifesting as nonspecific clinical symptoms (including pallor, fever, and anemia), and varying degrees of leukopenia and thrombocytopenia, hepatosplenomegaly being observed in up to one third of patients. The diagnosis of AEL is typically made within the first 3 months after a patient begins to report signs and symptoms. ${ }^{6,11}$ It is of note that, upon physical examination, our patient presented with no lymph node enlargement or organomegaly. However, mild splenomegaly was revealed by a CT scan of the abdomen. The autopsy revealed mild hepatosplenomegaly with leukemic infiltration, which also affected abdominal lymph nodes and other solid organs. This suggests that the tendency of neoplastic erythroblasts to involve solid organs is being underestimated, as has been reported by other authors. ${ }^{11}$ Also of note was the presence of myeloid metaplasia in the spleen and liver, a result of poor BM reserve. Myeloid metaplasia has been described in association with other BM diseases, especially myelofibrosis. ${ }^{12}$ We also found severe megakaryocyte atypia in the solid organs infiltrated by leukemia cells. Dysplastic megakaryocytes (or granulocytes) are more common and more significant in EL than in PEL. ${ }^{6}$

In cases of AEL, peripheral blood smears can show only cytopenias, with no evidence of blasts, which contributes to the difficulty in diagnosing the disease. Therefore, in order to establish a diagnosis of 

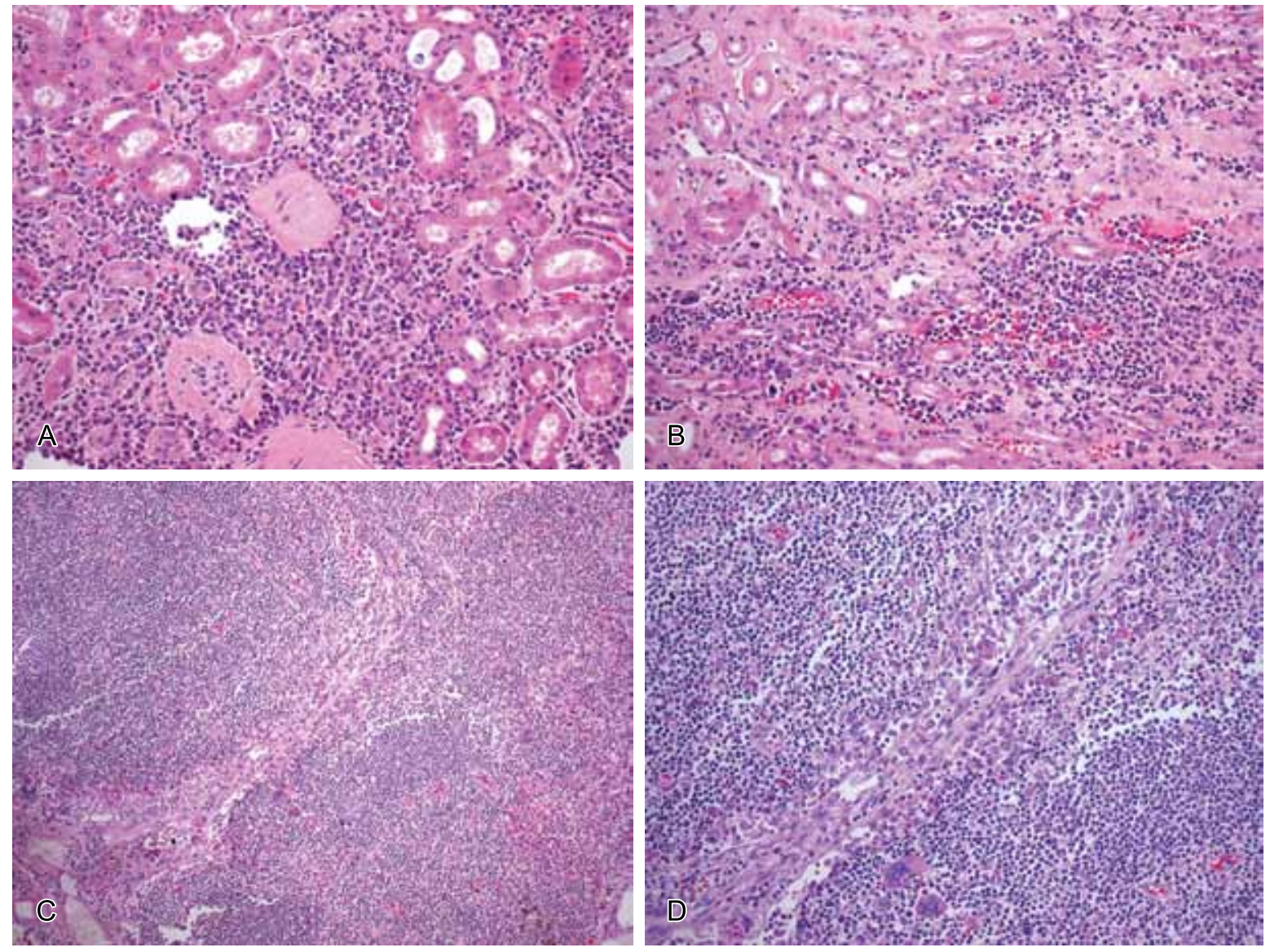

Figure 7 - Photomicrographs of the kidney and abdominal lymph node: A- renal parenchymal infiltration by immature myeloid cells (hematoxylin and eosin [H\&E]; magnification, $\times 200$ ); B- immature erythroid cells in the lumen of capillary vessels (H\&E; magnification, $\times 200$ ); C- lymph node infiltrated by immature erythroid cells, with atypical megakaryocytes in the subcapsular sinus (H\&E; magnification, $\times 100$ ); and D- immature erythroid cells and atypical megakaryocytes in the subcapsular sinus (H\&E; magnification, $\times 200$ ).

AEL, morphological examination of the BM specimens obtained by BM aspiration and biopsy, as well as a full panel of antibodies for leukemia immunophenotyping and exclusion of differential diagnoses, is needed. ${ }^{6}$ Immunophenotyping of AEL is best performed by flow cytometry of BM aspirates. However, in the case reported here, the diagnosis was established by immunohistochemical analysis of the BM biopsy specimen. This was due to a dry tap, which in turn was due to extensive necrosis. Immunohistochemistry reveals erythroblasts that are positive for glycophorin A and sometimes for CD117, similar to the normal pronormoblast population and the precursor cells of the granulocytic series in the BM. ${ }^{6}$ In cases of AEL, principally in those of the PEL subtype, the blasts are often negative for all erythroid-associated antibodies assessed by immunohistochemistry because many of the target antigens require some degree of erythroid maturation to be expressed, or a very dim expression may be detectable by immunohistochemistry. ${ }^{6}$
In the case reported here, immunohistochemical analysis of the BM biopsy specimen revealed an immature erythroid population with the following immunophenotype: glycophorin A-positive/CD117positive/CD34-negative/MPO-negative. The severe cytologic atypia, together with the formation of large cell aggregates, some of which were completely necrotic, were morphologically suggestive of metastatic epithelial cancer or high-grade non-Hodgkin's lymphoma. Those hypotheses were ruled out by negativity for epithelial and lymphoid markers, as determined by immunohistochemistry. The evaluation of the BM sample obtained during the autopsy, a sample with greater representation of the hematopoietic tissue, revealed a significant quantity of blasts of the granulocytic lineage, the phenotype of which was diffusely positive for MPO/CD117 and focally positive for CD34 ( $>20 \%$ of nonerythroid nucleated cells). Those findings met the criteria for a diagnosis of EL. The immunohistochemical analysis of solid organs, such as the liver and spleen, revealed low or absent expression of glycophorin $A$ in the immature erythroid 
cells (CD117-positive/MPO-negative), suggestive of undifferentiated neoplastic erythroblasts.

Liu et al. ${ }^{13}$ recently reported that, among the hematopoietic lineages, E-cadherin was apparently specific to the immature erythroid lineage, with weak to strong membrane positivity in most erythroblasts in PEL and only small positive groups in EL, suggesting that the association between the markers E-cadherin and glycophorin $A$ is useful in distinguishing between mature and immature precursors in BM biopsy specimens analyzed by immunohistochemistry. In the case reported here, the erythroid precursor population was not diffusely positive for E-cadherin. Few of the cells in the BM biopsy specimen were positive for E-cadherin, whereas all of the cells in the BM sample collected during autopsy were negative for E-cadherin. Maybe this finding supports the diagnosis of EL.

The differential diagnosis of AEL includes benign and malignant entities. As previously discussed, the diagnostic criteria for AEL established in the current $\mathrm{WHO}$ classification of $\mathrm{AML}^{1}$ include the need to rule out myelodysplastic syndromes (on the basis of patient history or cytogenetic abnormalities), erythropoietin therapy, and previous cancer treatment. In addition, in the presence of undifferentiated erythroblasts (as determined by morphological evaluation), the differential diagnosis should include lymphoma, myeloma/plasmacytoma, and other forms of leukemia, such as acute megakaryoblastic leukemia. Furthermore, cases of PEL are reported with partial expression of CD41 or CD61; whether these cases are better classified as acute mixed erythroid-megakaryoblastic leukemia or not is uncertain. ${ }^{6}$ A number of non-neoplastic diseases can cause erythroid predominance in the BM and therefore must be excluded before the diagnosis of AEL can be established; among the most common nonneoplastic disorders that need to be excluded before the diagnosis of $A E L$ is established is megaloblastic anemia due to nutritional deficiency of vitamin B12 or folate. ${ }^{1,6}$

In the case reported here, we believe that the combination of severe atypia with necrosis and numerous mitoses (as revealed by examination of the BM biopsy specimen) left no doubt as to the diagnosis of malignancy. The confirmation of the erythroid lineage and the exclusion of other lineages by the immunohistochemical panel were essential in order to establish the diagnosis. Although the dry tap prevented us from performing cytological examination of the BM aspirate, there were no clinical findings to warrant a relocation of the case to another diagnostic category, AEL being the most likely diagnosis.

In some cases, peripheral blood smears show only nonspecific cytopenias and not circulating blasts. Similarly, BM aspirate smears can be inconclusive because of hypocellularity, which is related to necrosis, cell fragility, or fibrosis. Therefore, in such cases, pathologists should establish the definitive diagnosis of AEL by examining the BM biopsy specimen. ${ }^{6}$ We emphasize the need for include the markers of hematologic lineages in malignancies with severe atypia in which the markers of primary metastatic sites are negative in BM samples and in samples from other organs.

The prognosis of AEL is unfavorable. The clinical behavior of the disease is aggressive, the mean reported survival ranging from 1.5 months to 19 months. ${ }^{10,11}$ The presence of cytogenetic abnormalities has recently been reported to correlate with clinical and biological characteristics, as well as with survival and treatment response. Patients with AEL with a complex karyotype have a worse prognosis..$^{10,14}$

Here, we described the BM biopsy findings and autopsy findings in an elderly female patient with a diagnosis of AEL of the EL subtype. Autopsy reports of AEL are extremely rare in the literature in English. ${ }^{11,15}$ The new, stricter, criteria established in the latest WHO classification of $\mathrm{AML}^{1}$ will certainly contribute to a deeper understanding of $A E L$ and allow future molecular biology studies to define molecular markers of AEL. We emphasize that the collection of a larger BM sample plays an important role in the diagnosis of $\mathrm{AEL}$, given that the disease can display an aggressive behavior, with infiltration of multiple organs, as observed in the case reported here.

\section{ACKNOWLEDGEMENTS}

We are grateful to Rosa Maria C. Zanardi for the technical support on the visual work.

\section{REFERENCES}

1. Arber DA, Brunning RD, Orazi A, et al. Acute myeloid leukemia, not otherwise specified. In: Swerdlow SH, Campo E, Harris NL, et al., editors. Tumors of haematopoietic and lymphoid tissues. Lyon: IARC Press; 2008. p.134-6. World Health Organization Classification of Tumors; vol. 2. 
2. Di Guglielmo G. Eritremie acute. Roma: Atti Congr. Ataliano Med. Int.; 1923.

3. Dameshk W, Baldini M. The di Guglielmo syndrome. Blood. 1958; 13:192-4. PMid:13510296.

4. Bennett JM, Catovsky D, Daniel MT, et al. Proposed revised criteria for the classification of acute myeloid leukemia: a report of the French-American-British Cooperative Group. Ann Inter Med. 1985; 103:620-5. PMid:3862359.

5. Bennett JM, Catovsky D, Daniel MT, et al. Proposal for classification of the acute leukemia: French-AmericanBritish co-operative Group. Br J Haematol. 1976; 33:451-8. PMid:188440. http://dx.doi.org/10.1111/j.1365-2141.1976. tb03563.x

6. Zuo Z, Polski JM, Kasyan A, Medeiros LJ. Acute erytroid leukemia. Arch Pathol Lab Med. 2010; 134:1261-70. PMid:20807044.

7. Mazzella FM, Kowal-Vern A, Shrit MA, et al. Acute erythroleukemia: evaluation of 48 cases with reference to classification, cell proliferation, cytogenetics, and prognosis. Am J Clin Pathol. 1998; 110:590-8. PMid:9802343.

8. Kowal-Vern A, Mazzella FM, Cotelingam JD, Shrit MA, Rector JT, Schumacher HR. Diagnosis and characterization of acute erythroleukemia subsets by determining the percentages of myeloblasts and proerythroblasts in 69 cases. Am J Hematol. 2000; 65:5-13. http://dx.doi.org/10.1002/10968652(200009)65:1\%3C5::AID-AJH2\%3E3.0.CO;2-U

9. Brunning RD, Matutes E, Flandrin G, et al. Acute myeloid leukemia not otherwise categorized. In: Jaffe ES, Harris $\mathrm{NL}$, Stein H, Vardiman JW, editors. Pathology and genetics of tumors of haematopoietic and lymphoid tissues. Lyon: IARC Press; 2001. p. 97-9. World Health Organization Classification of Tumors; vol. 3.

10. Kasyan A, Medeiros LJ, Zuo Z, Santos FP, et al. Acute erytroid leukemia as defined in the World Health Organization classification is a rare and pathogenetically heterogeneous disease. Mod Pathol. 2010; 23:1113-26. PMid:20473273. http://dx.doi.org/10.1038/modpathol.2010.96

11. Hasserjian RP, Howard J, Wood A, Henry K, Bain B. Acute erythremic myelosis (true etythroleukemia): a variant of AML FAB-M6. J Clin Pathol. 2001; 54:205-9. PMid:11253132. PMCid:1731380. http://dx.doi.org/10.1136/jcp.54.3.205

12. Abdel-Wahab OI, Levine RL. Primary myelofibrosis: update on definition, pathogenesis, and treatment. Annu Rev Med. 2009; 60:233-45. PMid:18947294. http://dx.doi. org/10.1146/annurev.med.60.041707.160528

13. Liu W, Hasserjian RP, Hu, Y, et al. Pure erytroid leukemia: a reassessment of the entity using the 2008 World Health Organization classification. Mod Pathol. 2011; 24:375-83. PMid:21102413. http://dx.doi.org/10.1038/modpathol.2010.194

14. Santos FPS, Faderl S, Garcia-Manero G, et al. Adult acute erythroleukemia: na analysis of 91 patients treated at a single institution. Leukemia. 2009; 23:2275-80. PMid:19741728. http://dx.doi.org/10.1038/leu.2009.181

15. Reiffers J, Bernard P, Larrue J, et al. Acute erythroblastic leukemia presenting as acute undifferentiated leukemia: a report of two cases with ultrastrutural features. Leuk Res. 1985; 9:413-20. http://dx.doi.org/10.1016/01452126(85)90064-5

\section{Conflict of interest: None}

Submitted on: $22^{\text {th }}$ October 2011

Accept on: $31^{\text {th }}$ October 2011

Correspondence: Serviço de Anatomia Patológica - Hospital Universitário USP

Av. Prof. Lineu Prestes, 2565, - Cidade Universitária - São Paulo/SP - Brazil

CEP: 05508-900 - Phone: +55 (11) 3091-9384

E-mail: crisrf@hu.usp.br 